Pesq. Vet. Bras. 35(4):344-348, abril 2015

DOI: $10.1590 / \mathrm{S} 0100-736 \mathrm{X000400005}$

\title{
Proteínas imunorreativas de Conidiobolus lamprauges isoladas de ovinos infectados naturalmente ${ }^{1}$
}

\author{
Maria C. Silva ${ }^{2,3}$, Isabela Godoy ${ }^{3}$, Daniel G. Ubiali ${ }^{4}$, Marcelo M. Silveira ${ }^{3}$, Letícia \\ C. Pitchenin ${ }^{3}$, Laila N.S. Brandão ${ }^{3}$, Valéria Dutra ${ }^{3 *}$ e Luciano Nakazato ${ }^{3}$
}

\begin{abstract}
Silva M.C., Godoy I., Ubiali D.G., Silveira M.M., Pitchenin L.C., Brandão L.N.S., Dutra V. \& Nakazato L. 2015. [Immunoreactive proteins of Conidiobolus lamprauges isolated from naturally infected sheep.] Proteínas imunorreativas de Conidiobolus lamprauges isoladas de ovinos infectados naturalmente. Pesquisa Veterinária Brasileira 35(4):344-348. Departamento de Clínica Médica Veterinária, Faculdade de Agronomia, Medicina Veterinária e Zootecnia, Universidade Federal de Mato Grosso, Av. Fernando Corrêa da Costa 2673, Bairro Boa Esperança, Cuiabá, MT 78068-900, Brazil. E-mail: valdutra@ufmt.br

The study of sheep conidiobolomycosis has been carried out in its clinical, epidemiological, pathological and molecular aspects. Information, however, about the host immune response in infection Conidiobolus lamprauges is absent. This study aimed to identify immunoreactive proteins that may play an important role in the immune response of sheep naturally infected by C. lamprauges. For protein and immunological characterization, C. lamprauges (strain FIOCRUZ-INCQS 40316) isolated from a sheep with clinical signs of conidiobolomycosis in the MT state and five sera samples of naturally infected sheep were used. The presence of IgG antibody was observed in all patients with reagent titers in dilutions up to 1:1600. In immunoblot technique, the antigenic profile against infected sheep sera showed twelve reactive bands with molecular weights ranging from 35 to $198 \mathrm{kDa}$. Among them, the $198 \mathrm{kDa}$ protein was reactive against sera from three sheep and the 53 $\mathrm{kDa}$ showed increased intensity compared to other bands probably being immunodominant. Healthy animal serum samples showed no reactivity demonstrating the specificity of the technique. The presence of antigenic proteins of $C$. lamprauges and specific IgG in sheep sera observed in this study may assist in the development of early diagnostic methods and the use of protein as candidate vaccines for the control and prevention of infection in animals and human.
\end{abstract}

INDEX TERMS: Conidiobolus lamprauges, sheep, immunogenic antigen.

RESUMO.- 0 estudo de conidiobolomicose ovina tem sido realizado nos seus aspectos clínicos, epidemiológicos, patológicos e moleculares. Informações, entretanto, sobre a resposta imune do hospedeiro na infecção por Conidiobolus lamprauges são inexistentes. Este estudo teve por objetivo

\footnotetext{
${ }^{1}$ Recebido em 3 de setembro de 2014.

Aceito para publicação em 12 de abril de 2015.

${ }^{2}$ Departamento de Ensino do Curso Técnico em Alimentos, Instituto Federal de Educação, Ciência e Tecnologia de Mato Grosso, Campus Barra do Garças, Estrada de Acesso a BR-158 s/n, Barra do Garças, MT 78600-000, Brasil.

${ }^{3}$ Programa de Pós-Graduação em Ciências Veterinárias, Universidade Federal de Mato Grosso (UFMT), Av. Fernando Corrêa da Costa 2367, Bairro Boa Esperança, Cuiabá, MT 78060-900, Brasil. *Autor para correspondência: valdutra@ufmt.br

${ }^{4}$ Setor de Patologia Veterinária, UFMT, Av. Fernando Corrêa da Costa 2367, Bairro Boa Esperança, Cuiabá, MT 78060-900.
}

a identificação de proteínas imunorreativas que possam desempenhar papel importante na resposta imune de ovinos naturalmente infectados por $C$. lamprauges. Para a caracterização protéica e imunológica foi utilizada a cepa de C. lamprauges (FIOCRUZ-INCQS 40316) isolada de ovino com sinais clínicos de conidiobolomicose no Estado do MT e cinco amostras de soro de ovinos infectados naturalmente pelo fungo. A presença de anticorpos IgG foi observada em todos os animais doentes com títulos reagentes em diluições de até 1:1.600. Na técnica do immunoblot, o perfil antigênico frente aos soros ovinos com a doença apresentou doze bandas reativas, com massas moleculares variando de 35 a 198 kDa. Dentre estas, a proteína de 198 kDa foi reativa em 3 soros de ovinos e a de $53 \mathrm{kDa}$ apresentou a maior intensidade comparativamente com outras bandas, sendo provavelmente imunodominante. Amostras de soro 
de animais sadios não apresentaram reatividade demostrando a especificidade da técnica. A presença de proteínas antigênicas de $C$. lamprauges e IgG específicos em soros de ovinos observados no presente trabalho poderá auxiliar no desenvolvimento de métodos de diagnóstico precoces e na utilização de proteínas candidatas a vacinas para o controle e prevenção da infecção em animais e humanos.

TERMOS DE INDEXAÇÃO: Conidiobolus lamprauges, ovinos, antígenos imunorreativos.

\section{INTRODUÇÃO}

Zigomicoses invasivas são causadas por fungos das ordens Mucorales e Entomophthorales. Dentro dessa última, os fungos saprófitos do gênero Conidiobolus (C.) têm importância médica e veterinária e estão frequentemente associados com rinite granulomatosa crônica no homem e em várias espécies animais (Humber et al. 1989, Zamos et al. 1996, Bauer et al. 1997, Tadano et al. 2005, Kimura et al. 2011, Isa-Isa et al. 2012), sendo Conidiobolus coronatus, C. incongruus e $C$. lamprauges as principais espécies patogênicas (Humber et al. 1989, Carrigan et al. 1992, Ribes et al. 2000, Vilela et al. 2010). A conidiobolomicose vem ganhando importância pela dificuldade no tratamento em função da baixa resposta terapêutica à maioria dos antifúngicos disponíveis (Boabaid et al. 2008, Kimura et al. 2011, Tondolo et al. 2013).

A enfermidade na espécie ovina vem ocorrendo em países de clima tropical e subtropical (Carrigan et al. 1992, Ketterer et al. 1992, Morris et al. 2001, Silva et al. 2007a). No Brasil, casos da doença já foram identificados nos Estados do Piauí, Paraíba, Mato Grosso, Rio Grande do Sul e Santa Catarina, sendo descritos como um problema sanitário grave acarretando perdas econômicas consideráveis aos produtores de ovinos nessas regiões (Silva et al. 2007a, 2007b, Riet-Correa et al. 2008, Boabaid et al. 2008, Pedroso et al. 2009, De Paula et al. 2010, Furlan et al. 2010) com taxa de letalidade de 100\% (Silva et al. 2007b). Nos Estados do Piauí, Mato Grosso e Santa Catarina a etiologia da doença está associada à infecção por $C$. lamprauges (Silva et al. 2007a, De Paula et al. 2010, Furlan et al. 2010, Vilela et al. 2010).

O diagnóstico da doença tem como princípio os achados epidemiológicos, clínico-patológicos associados ao isolamento do agente em cultivo com a classificação definitiva realizada através de técnicas moleculares como a PCR (Prophet et al. 1992, Ribes et al. 2000, Riet-Correa et al. 2008, Vilela et al. 2010, De Paula et al. 2010, Ubiali et al. 2013, Silveira et al. 2013).

A patogenia da conidiobolomicose não está esclarecida. Genes associados à virulência foram estudados na infecção do fungo em insetos (Freimoser et al. 2003) e a sua capacidade de termo resistência em diferentes temperaturas (Silva et al. 2012, Godoy et al. 2014).

Dados de resposta imunológica do hospedeiro não foram descritos até o momento. Desta maneira, este estudo teve por objetivo a identificação de proteínas imunorreativas que possam desempenhar papel importante na resposta imune de ovinos naturalmente infectados por $C$. lamprauges.

\section{MATERIAL E MÉTODOS}

Cepa de referência. No presente estudo, foi utilizada a cepa de Conidiobolus lamprauges M290/07 (FIOCRUZ-INCQS 40316), isolada de ovino com conidiobolomicose oriundo do município de Nobres/MT, para a caracterização protéica e imunológica. 0 isolado foi mantido em meio Agar Sabouraud Dextrose (SDA) 2\% a $37^{\circ} \mathrm{C}$.

Amostras de soro. Cinco amostras de soro (A, B, C, D e E) foram coletadas de ovinos com sinais clínicos e diagnóstico confirmado de conidiobolomicose de quatro diferentes municípios do Estado de Mato Grosso (Quadro 1). A doença foi confirmada por isolamento e PCR. Como controle negativo foram utilizadas duas amostras de ovinos saudáveis (F e G). As amostras de sangue foram coletadas por venopunção da jugular em tubos sem anticoagulante, centrifugadas, separadas e os soros estocados a $-20^{\circ} \mathrm{C}$.

Extração de Proteínas de Conidiobolus lamprauges. Para obtenção da proteína, o isolado INCQS 40316 foi cultivado sobre papel filtro em meio SDA e incubado a $37^{\circ} \mathrm{C}$ durante cinco dias. A extração de proteínas foi realizada segundo adaptações do protocolo descrito por Lakshman et al. (2008). A quantificação das amostras foi realizada pelo método colorimétrico de Bradford (1976).

Ensaio imunoenzimatico (ELISA). A técnica foi realizada de acordo com a metodologia descrita por Santurio et al. (2006) com modificações. Placas de poliestireno de 96 cavidades (BD- Biosciences) foram sensibilizadas com $1 \mu \mathrm{g} /$ poço de extrato protéico diluído em tampão carbonato-bicarbonato 0,05M (pH 9,6) e incubadas em câmara úmida "overnight" a $4^{\circ} \mathrm{C}$. Posteriormente, as placas foram submetidas a três lavagens com PBS-T $1137 \mathrm{mM}$ $\mathrm{NaCl} ; 2,7 \mathrm{mM} \mathrm{KCl} ; 10 \mathrm{mM} \mathrm{Na}_{2} \mathrm{HPO}_{4} ; 2 \mathrm{mM} \mathrm{KH}_{2} \mathrm{PO}_{4}$; água destilada qsp $1000 \mathrm{ml}$; pH 7,4; 0,1\% Tween 20) seguida de incubação com $100 \mu \mathrm{L}$ tampão PBS-TB (PBS-T acrescido de leite em pó desnatado a $5 \%$ ) e incubadas a $37^{\circ} \mathrm{C}$ por uma hora. Após um novo ciclo de três lavagens das placas com PBS-T, as mesmas foram incubadas por uma hora a $37^{\circ} \mathrm{C}$ com anticorpos primários em diluições seriadas (1:200, 1:400, 1:800 e 1:1600) PBS-T. Foram realizadas três lavagens com PBS-T seguidas da adição de $100 \mu \mathrm{L}$ de anticorpos secundários anti-IgG ovino conjugado com peroxidase (Sigma) na diluição de 1:2000 e incubado durante uma hora a $37^{\circ} \mathrm{C}$. Em seguida realizaram-se três lavagens com PBS-T, e adicionou-se $100 \mu \mathrm{L}$ do substrato cromógeno 3,3,5,5 tetramethylbenzidine (TMB) por 5 min a temperatura ambiente. A leitura das amostras foi realizada a $595 \mathrm{~nm}$, em duplicatas, no espectrofotômetro Epoch (Biotek).

Eletroforese em Gel de Poliacrilamida (SDS-PAGE) e Immunoblot. Para a realização da eletroforese, $50 \mu \mathrm{g}$ de extrato protéico total foi precipitado com solução de ácido tricloroacético (TCA): acetona (1:9), seguido de incubação a $-20^{\circ} \mathrm{C}$, por $30 \mathrm{~min}$ e centrifugação a $12.600 \mathrm{~g}$ durante $20 \mathrm{~min}$. 0 precipitado formado foi lavado com $1 \mathrm{~mL}$ de acetona e ressuspendido em $100 \mu \mathrm{L}$ de tampão de amostra desnaturante $1 \mathrm{X}$ (Tris- $\mathrm{HCl}$ 0,5M pH 6,8, gli-

Quadro 1. Descrição das amostras de soro ovino utilizados no estudo

\begin{tabular}{lllcc}
\hline Soro & \multicolumn{1}{c}{ Município } & Diagnóstico & \multicolumn{2}{c}{ Forma clínica } \\
\cline { 3 - 4 } & & & Rinofacial & Rinofaríngea \\
\hline A & Poconé & C. lamprauges & + & \\
B & Barão Melgaço & C. lamprauges & + & \\
C & Alto Paraguai & C. lamprauges & + & \\
D & Nobres & C. lamprauges & & + \\
E & Nobres & C. lamprauges & & + \\
F & Sto A. do Leverger & $\bullet$ & . & - \\
G & Sto A. do Leverger & $\bullet$ & & \\
\hline
\end{tabular}


cerol 10\%, 2-mercaptoetanol 4\%, SDS $2 \%$ e azul de bromofenol $0,01 \%$ ) seguido de homogeneização e incubação a $100^{\circ} \mathrm{C}$ por 5 minutos. A separação protéica foi realizada em gel de poliacrilamida segundo Laemmli (1970). Os géis foram submetidos à coloração de Coomassie "overnight" e descorados em solução descolorante de acordo com Ausubel et al. (2003).

Após a eletroforese, as proteínas foram transferidas do gel para uma membrana de PVDF (fluoreto de polivinilideno) (Hybond-P, GE Heathcare), conforme instruções do fabricante. As membranas foram bloqueadas com PBS-TB e submetidas à lavagem com PBS-T a temperatura ambiente por 1 hora e 5 minutos, respectivamente. A membrana foi incubada com os soros dos diferentes animais (Quadro 1). Após essa etapa, as membranas foram lavadas duas vezes com PBS-T por 10 min, seguida de incubação por 1 hora em anticorpo secundário Anti-IgG de carneiro marcado com peroxidase (Sigma) diluído 1:2000. Após esse período, foram submetidas a três ciclos de lavagens de 10 min com PBS-T. Após a retirada do excesso de PBS-T, foi adicionada à membrana 0 cromógeno TMB com interrupção da reação com ácido sulfúrico $1 \mathrm{M}$. Todas as lavagens e incubações foram realizadas à temperatura ambiente e todas as capturas de imagens realizadas no sistema ChemiDoc ${ }^{\mathrm{Tm}} \mathrm{XRS}+$ (Bio-Rad) com auxílio do software Image $\mathrm{Lab}^{\mathrm{Tm}}$ (Bio-Rad).

\section{RESULTADOS}

Na quantificação das proteínas pelo método de Bradford (1976) o rendimento do extrato obtido foi de $7 \mu \mathrm{g} / \mu \mathrm{L}$. A análise de $50 \mu \mathrm{g}$ do extrato protéico de Conidiobolus lamprauges por SDS-PAGE corado com Coomassie permitiu evidenciar presença de bandas com massas moleculares variando de 6 a 198 kDa (Fig.1).

Em relação à presença de anticorpos específicos, todas as amostras de soros de animais doentes foram reativas. Os títulos de anticorpos para proteínas de C. lamprauges foram observados em diluições variando de 1:100 a 1:1.600 (Fig.2). Para estabelecer o ponto de corte da reação foi considerada a média dos valores de leitura de densidade ótica

\section{$\begin{array}{llllllll}1 & A & B & C & D & E & F & G\end{array}$}

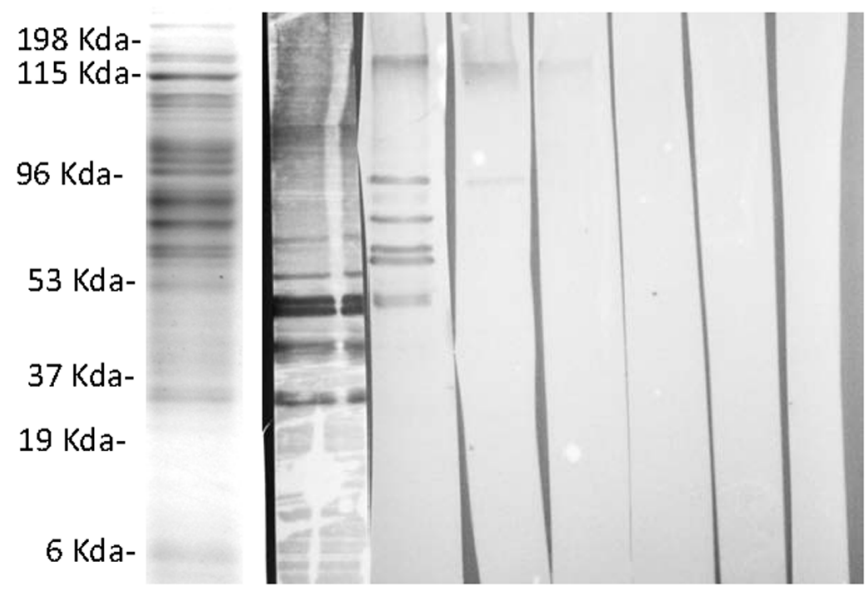

Fig.1. Perfil protéico de Conidiobolus lamprauges (INCQS 40316) de micélio em gel de poliacrilamida (SDS-PAGE 12\%) e imunoblot com soro de ovinos doentes por C. lamprauges. (1) Extrato protéico de C. lamprauges (INCQS 40316); (A-E) Soro de ovinos naturalmente infectados; $(F, G)$ Soro de ovinos saudáveis.

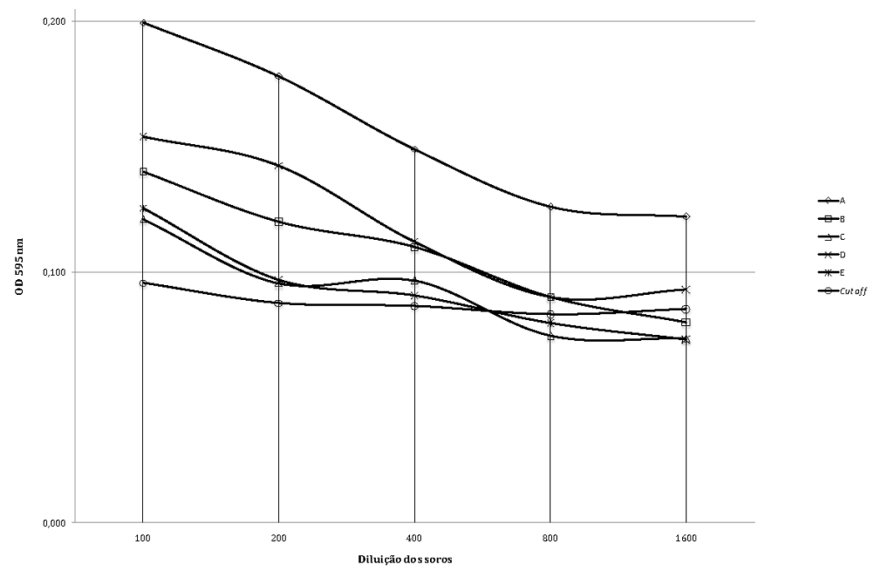

Fig.2. Títulos médios de duplicatas das amostras de soro de ovinos naturalmente infectados com Conidiobolus lamprauges (A-E) e saudáveis $(F, G)$ por ELISA.

(OD) das amostras negativas acrescidas de duas vezes o desvio padrão (Fig.2). Os soros apresentaram positividade até diluições de 1:400. Somente a amostra do animal A, entretanto, apresentou títulos altos com positividade em diluições de 1:800 e 1:1600 (Fig.2).

Durante a realização do Immunoblot, o perfil antigênico resultante da reação do extrato protéico de $C$. lamprauges frente aos soros de ovinos com a enfermidade apresentou doze bandas reativas. As massas moleculares destas foram $198,115,96,90,80,75,70,65,60,53,50$ e $35 \mathrm{kDa}$ em quatro amostras de soros (A, B, C e D). Algumas bandas foram reativas apenas para um dos soros testados (bandas de $115,75,60,50$ e $35 \mathrm{kDa}$ na amostra $\mathrm{A}$, e as de $90,80,70$ e $65 \mathrm{kDa}$ na amostra B). Duas proteínas reagiram de maneira similar para dois soros (96kDa para os soros B e C e $53 \mathrm{kDa}$ para os soros A e B). A proteína de $198 \mathrm{kDa}$ reagiu com três soros (B, C e D). 0 soro E não reagiu com nenhuma das proteínas. Da mesma forma, os soros utilizados como controle negativos ( $\mathrm{F}$ e $\mathrm{G}$ ) não apresentaram reatividade frente às proteínas extraídas de C. lamprauges (Fig.1).

\section{DISCUSSÃO}

A análise do perfil protéico do extrato miceliano submetido à separação por SDS-PAGE permitiu visualizar o padrão de bandas do isolado de Conidiobolus lamprauges sendo evidenciadas bandas em toda extensão do gel, com massas moleculares variando de 6 a $198 \mathrm{kDa}$. A diversidade de bandas observadas neste estudo difere do observado em estudo prévio realizado por Mendonza et al. (1992) utilizando outra espécie, $C$. coronatus, onde os mesmos evidenciaram pela coloração de prata 16 bandas, nove de alto peso molecular (97 a $31 \mathrm{kDa}$ ) e sete com massa molecular inferior a $31 \mathrm{kDa}$. A diferença no número de bandas observadas neste estudo pode estar relacionada a diferenças entre as espécies utilizadas, as condições de cultivo do fungo e do preparo dos antígenos (Camargo \& Franco 2000).

No teste de ELISA foi possível detectar anticorpos anti-extratos protéicos de $C$. lamprauges. Os níveis de detecção de anticorpos variaram conforme o soro testado. A maior reatividade da amostra $\mathrm{A}$ e a diferença nos títulos obser- 
vados podem estar relacionadas à resposta dos animais frente à infecção por $C$. lamprauges. Em algumas infecções fúngicas, como a cromoblastomicose humana, os títulos de anticorpos variam muito entre pacientes doentes (Esterre et al. 2000) e, em casos de outros fungos como Coccidioides immitis em equinos, os títulos mais altos estão associados a quadros clínicos com prognósticos desfavoráveis, geralmente associados à pneumonia e infecções generalizadas (Higgins et al. 2007).

O ELISA vem sendo utilizado na detecção de anticorpos específicos nos casos de pitiose, mostrando alto grau de sensibilidade e especificidade, possibilitando a detecção de infecções na fase inicial da doença (Mendoza et al. 1997, Santurio et al. 2006). Mendoza et al. (1992) em trabalho conduzido com amostras de cinco soros obtidos de equinos com pitiose identificaram um antígeno de $44 \mathrm{kDa}$, que reagiu de forma cruzada com antígeno de $C$. coronatus, não sendo esclarecida a relação antigênica entre as espécies. Em nosso estudo a utilização do extrato protéico de cultivo não apresentou reatividade com amostras de ovinos com pitiose, sendo possível a utilização desta técnica de forma específica para o diagnóstico de ovinos com conidiobolomicose.

Os anticorpos presentes nos soros detectaram doze proteínas antigênicas para $C$. lamprauges com massas moleculares estimadas de 198, 115, 96, 90, 80, 75, 70, 65, 60, 53, 50 e $35 \mathrm{kDa}$. Em uma amostra não se verificou reconhecimento de proteínas antigênicas para caracterização imunológica, porém, foram observadas variações no número e na intensidade de bandas em quatro soros reagentes. Houve diferença no padrão de reconhecimento entre os casos rinofaciais e rinofaríngeos da doença. A variação na resposta imune humoral observada pode estar relacionada à virulência da cepa, à fase de infecção e à cronicidade da doença para o desencadeamento de uma resposta imune do hospedeiro (Krajaejun et al. 2006, Zapata et al. 2012).

Dentre as proteínas observadas no estudo, as de massas moleculares 53 e $198 \mathrm{kDa}$ parecem ser imunodominantes, destacando-se das demais, pela maior intensidade da reação. Portanto, a identificação e caracterização das mesmas podem contribuir para o entendimento dos mecanismos de patogenicidade nos casos da conidiobolomicose ovina, bem como na utilização para diagnósticos sorológicos ou como candidatos a vacinas. Estudos conduzidos em fungos patogênicos de importância biomédica já permitiram obter importantes informações sobre proteínas imunogênicas com papel fundamental no processo de invasão, sobrevivência no hospedeiro e como possíveis candidatos a vacina (Camargo et al. 1989, Krajaejun et al. 2006, Chindamporn et al. 2009, Perenha-Viana et al. 2012, Magalhães et al. 2012).

\section{CONCLUSÃO}

A presença das proteínas antigênicas observadas no presente trabalho poderá auxiliar no entendimento dos mecanismos patogênicos e no papel na imunidade do hospedeiro, além de contribuir para o desenvolvimento de métodos de diagnóstico precoces e estabelecimento de tratamentos clínicos e vacinas pela importância crescente de Conidiobolus lamprauges na medicina veterinária e humana.
Agradecimentos.- À Fundação de Amparo à Pesquisa do Estado de Mato Grosso (Fapemat) e a CAPES pela bolsa concedida.

\section{REFERÊNCIAS}

Ausubel F.M., Brent R., Kingston R.E., Moore D.D., Seidman J.G., Smith J.A. \& Stuhl K. 2003. Current Protocols in Molecular biology. Copyright John Weley and Sons. New York, NY. 4410p.

Bauer R.W., Lemarie S.L. \& Roy A.F. 1997. Oral conidiolomycosis in a dog. Vet. Dermatol. 8(2):115-120.

Boabaid F.M., Ferreira E.V., Arruda L.P., Gasparetto N.D., Souza R.L., Silva M.C., Dutra V., Nakazato L. \& Colodel E.M. 2008. Conidiobolomicose em ovinos no Estado de Mato Grosso. Pesq. Vet. Bras. 28(1):77-81.

Bradford M.M. 1976. A rapid and sensitive method for the quantification of microgram quantities of protein utilizing the principle of protein-dye binding. Anal. Biochem. 72:248-254.

Camargo Z.P. \& Franco M.F. 2000. Current knowledge on pathogenesis and immunodiagnosis of paracoccidioidomycosis. Revta Iberoam. Micol. $17: 41-48$.

Camargo Z.P., Unterkircher C. \& Travassos L.R. 1989. Identification of antigenic polypeptides of Paracoccidioides brasiliensis by immunoblotting. J. Med. Vet. Mycol. 27:407-412.

Carrigan M.J., Small A.C. \& Perry G.H. 1992. Ovine nasal zygomycosis caused by Conidiobolus incongruous. Aust. Vet. J. 69(10):237-240.

Chindamporn A., Vilela R., Hoag K.A. \& Mendoza L. 2009. Antibodies in the sera of host species with pythiosis recognize a variety of unique immunogens in geographically divergent Pythium insidiosum strains. Clin. Vaccine Immunol. 16(3):330-336.

De Paula D.A., Oliveira Filho J.X., Silva M.C., Colodel E.M., Broetto L., Pinto P.M., Schrank A., Nakazato L. \& Dutra V. 2010. Molecular characterization of ovine zygomycosis in central western Brazil. J. Vet. Diagn. Invest. 22:274-277.

Esterre P., Jahevitra M. \& Andriantsimahavandy A. 2000. Humoral immune response in chromoblastomycosis during and after therapy. Clin. Diagn. Lab. Immunol. 7(3):497-500.

Freimoser F., Screen S., Hu G. \& Leger R. 2003. EST analysis of genes expressed by the zygomycete pathogen Conidiobolus coronatus during growth on insect cuticle. Microbiology 149(7):1893-1900.

Furlan H.F., Lucioli J., Veronezi L.O., Fonteque J.H., Traverso S.D., Nakazato L. \& Gava A. 2010. Conidiobolomicose causada por Conidiobolus lamprauges em ovinos no Estado de Santa Catarina. Pesq. Vet. Bras. 30(7):529-532.

Godoy I., De Paula D.A.J., Silveira M.M., Brandão L.N.S., Dutra V. \& Nakazato L. 2014. Expressão diferencial de proteínas do fungo Conidiobolus lamprauges cultivado em diferentes temperaturas. Ciência Rural 44(3):473478.

Higgins J.C., Pusterla N. \& Pappagianis D. 2007. Comparison of Coccidioides immitis serological antibody titres between forms of clinical coccidioidomycosis in horses. Vet. J. 173:118-123.

Humber R.A., Brown C.C. \& Kornegay R.W. 1989. Equine zygomycosis caused by Conidiobolus lamprauges. J. Clin. Microbiol. 27(3):573-576.

Isa-Isa R., Arenas R., Fernandez R.F. \& Isa M. 2012. Rhinofacial conidiobolomycosis (entomophthoramycosis). Clin. Dermat. 30:409-412.

Ketterer P.J., Kelly M.A., Connole M.D. \& Ajello L. 1992. Rhinocerebral and nasal zygomycosis in sheep caused by Conidiobolus incongruous. Aust. Vet. J. 69(4):85-87.

Kimura M., Yaguchi T., Sutton D.A., Forthergill A.W., Thompson E.H. \& Wickes B.L. 2011. Disseminated human connidiobolomycosis due to Conidiobolus lampraugeus. J. Clin. Microbiol. 49:752-756.

Krajaejun T., Kunakorn M., Pracharktam R., Chongtrakool P., Sathapatayavongs B., Chaiprasert A., Vanittanakom N., Chindampom A. \& Mootsikapun P. 2006. Identification of a novel 74-kiloDalton immunodominant antigen of Pythium insidiosum recognized by sera from human patients with pythiosis. J. Clin. Microbiol. 44(5):1674-1680.

Laemmli U.K. 1970. Cleavage of structural proteins during the assembly of the head bacteriophage T4. Nature 227(259):680-685. 
Lakshman D.K., Natarajan S.S., Lakshman S., Garrett W.M. \& Dhar A.K. 2008. Opitimized protein extraction methods for proteomic analysis of Rhizoctonia solari. Mycologia 100(6):867-875.

Magalhães A., Ferreira K.S., Almeida S.R., Nosanchuk J.D., Travassos L.R. \& Taborda C.P. 2012. Prophylactic and therapeutic vaccination using dendritic cells primed with peptide 10 derived from the 43-kilodalton glycoprotein of Paracoccidioides brasiliensis. Clin. Vaccine Immunol. 19(1):23-29.

Mendoza L., Kaufman L. \& Mandy W.G.R. 1997. Serodiagnosis of human and animal pythiosis using an enzyme-linked immunosorbent assay. Clin. Diag. Lab. Immunol. 4(6):715-718.

Mendoza L., Nicholson V. \& Prescott J.F. 1992. Immunoblot analysis of the humoral immune response to Pythium insidiosum in horses with pythiosis. J. Clin. Microbiol. 30(11):2980-2983.

Morris M., Ngeleka M., Adogwa A.O., Lalla G., St-Germanin G. \& Higgins R. 2001. Rhinocerebral zygomycosis in a sheep. Can. Vet. J. 42:227-228.

Pedroso P., Raymundo D., Bezerra J., Oliveira E., Sonne L., Dalto A. \& Driemeier D. 2009. Rinite micótica rinofaríngea em um ovino Texel no Rio Grande do Sul. Acta Scient. Vet. 37:181-185.

Perenha-Viana M.C.Z., Gonzales I.A.A., Brockelt S.R., Machado L.N.C. \& Svidzinski T.I.E. 2012. Serological diagnosis of Paracoccidioidomycosis through a Western blot technique. Clin. Vaccine Immunol. 19(4):616619.

Prophet E.B., Mills B., Arrington J.B. \& Sobin L.H. 1992. Laboratory Methods in Histotechnology. Armed Forces Institute of Pathology, Washington. $279 \mathrm{p}$.

Ribes J., Vanover-Sams C.L. \& Baker D.J. 2000. Zygomycetes in human disease. Clin. Microbiol. Rev. 13:236-301.

Riet-Correa F., Dantas A.F.M., Azevedo E.O., Simões S.D.V., Silva S.M.M.S., Vilela R. \& Mendoza L. 2008. Outbreaks of rhinofacial and rhinopharyngeal zygomycosis in sheep in Paraíba, northeastern Brazil. Pesq. Vet. Bras. 28(1):29-35.

Santurio J.M., Leal A.T., Leal A.B.M., Alves S.H., Lubeck I., Griebeler J. \& Copetti M.V. 2006. Teste de Elisa indireto para diagnostico sorológico de pitiose. Pesq. Vet. Bras. 26:47-50.
Silva J.A.G., De Paula D.A.J., Silveira M.M., Silva M.C., Brandao L.N.S., Pitchenin L.C., Nakazato L. \& Dutra V. 2012. Genes termorregulados diferencialmente expressos em Conidiobolus lamprauges. Ciência Rural 42(9):1610-1613.

Silva S.M.M.S., Castro R.S., Costa F.A.L., Vasconcelos A.C., Batista M.C.S., Riet-Correa F. \& Carvalho E.M.S. 2007a. Conidiobolomycosis in sheep in Brazil. Vet. Pathol. 44:314-319.

Silva S.M.M.S., Castro R.S., Costa F.A.L., Vasconcelos A.C., Batista M.C.S., Riet-Correa F., Carvalho E.M.S. \& Lopes J.B. 2007b. Epidemiologia e sinais clínicos da conidiobolomicose em ovinos no Estado do Piauí. Pesq. Vet. Bras. 27(4):184-190.

Silveira M.M., De Paula D.A.J., Silva M.C., Pitchenin L.C., Cruz R.A.S., Colodel E.M., Dutra V. \& Nakazato L. 2013. Development and application of polymerase chain reaction test for detection of Conidiobolus lamprauges. Pesq. Vet. Bras. 33(12):1448-1452.

Tadano T., Paim N.P., Hueb M. \& Fontes C.J.F. 2005. Entomoftoromicose (zigomicose) causada por Conidiobolus coronatus in Mato Grosso (Brasil): relato de caso. Rev. Soc. Bras. Med. Trop. 38 (2):188-1990.

Tondolo J.S.M., Loreto E.S., Dutra V., Nakazato L., De Paula D.A.J., Zanette R.A., Alves S.H. \& Santurio J.M. 2013. In vitro susceptibility of Conidiobolus lamprauges recovered from sheep to antifungal agents. Vet. Microbiol. 166 (3/4):690-693.

Ubiali D.G., Cruz R.A.S., De Paula D.A.J., Silva M.C., Mendonça F.S., Dutra V., Nakazato L., Colodel E.M. \& Pescador C.A. 2013. Pathology of nasal infection caused by Conidiobolus lamprauges and Pythium insidiosum in sheep. J. Comp. Pathol. 149(2/3):137-145.

Vilela R., Silva S.M.S., Riet-Correa F., Dominguez E. \& Mendonza L. 2010. Morphologic and phylogenetic characterization of Conidiobolus lamprauges recovered from infected sheep. J. Clin. Microbiol. 48(2):427432.

Zamos D.T., Shumacher J. \& Loy J.K. 1996. Nasopharyngeal conidiobolomycosis in a horse. J. Am. Vet. Med. Assoc. 208(1):100-101.

Zapata F., Perazzo Y., Ortega I., Torres A. \& Lopez J.A. 2012. Caracterizacion de proteínas inmunodominantes de Pythium insidiosum y su uso en inmunoensayos para la deteccion de pitiosis em perro (Canis lupus familiaris). Gac. Cienc. Vet. 17(1):25-30. 